\title{
EEG-Guided Transcranial Magnetic Stimulation Reveals Rapid Shifts in Motor Cortical Excitability during the Human Sleep Slow Oscillation
}

\author{
Til O. Bergmann, ${ }^{1,2}$ Matthias Mölle, ${ }^{3}$ Marlit A. Schmidt, ${ }^{1}$ Christoph Lindner, ${ }^{1}$ Lisa Marshall, ${ }^{3}$ Jan Born, ${ }^{3,4}$ \\ and Hartwig R. Siebner ${ }^{1,5,6}$ \\ ${ }^{1}$ Department of Neurology, Christian-Albrechts University of Kiel, 24105 Kiel, Germany, ${ }^{2}$ Donders Institute for Brain, Cognition, and Behavior, Radboud \\ University Nijmegen, 6500 HB Nijmegen, The Netherlands, ${ }^{3}$ Department of Neuroendocrinology, University of Lübeck, 23538 Lübeck, Germany, ${ }^{4}$ Institute \\ of Medical Psychology and Behavioral Neurobiology, University of Tübingen, 72074 Tübingen, Germany, ${ }^{5}$ Danish Research Center for Magnetic Resonance, \\ Copenhagen University Hospital Hvidovre, 2650 Hvidovre, Denmark, and 'Institute of Neurology, Psychiatry and Senses, University of Copenhagen, 2200 \\ Copenhagen, Denmark
}

Evoked cortical responses do not follow a rigid input- output function but are dynamically shaped by intrinsic neural properties at the time of stimulation. Recent research has emphasized the role of oscillatory activity in determining cortical excitability. Here we employed EEG-guided transcranial magnetic stimulation (TMS) during non-rapid eye movement sleep to examine whether the spontaneous $<1 \mathrm{~Hz}$ neocortical slow oscillation (SO) is associated with corresponding fluctuations of evoked responses. Whereas the SO's alternating phases of global depolarization (up-state) and hyperpolarization (down-state) are clearly associated with fluctuations in spontaneous neuronal excitation, less is known about state-dependent shifts in neocortical excitability. In 12 human volunteers, single-pulse TMS of the primary motor cortical hand area $\left(\mathrm{Ml}_{\mathrm{HAND}}\right)$ was triggered online by automatic detection of SO up-states and down-states in the EEG. Statedependent changes in cortical excitability were traced by simultaneously recording motor-evoked potentials (MEPs) and TMS-evoked EEG potentials (TEPs). Compared to wakefulness and regardless of SO state, sleep MEPs were smaller and delayed whereas sleep TEPs were fundamentally altered, closely resembling a spontaneous SO. However, both MEPs and TEPs were consistently larger when evoked during SO up-states than during down-states, and ampliudes within each SO state depended on the actual EEG potential at the time and site of stimulation. These results provide first-time evidence for a rapid state-dependent shift in neocortical excitability during a neuronal oscillation in the human brain. We further demonstrate that EEG-guided temporal neuronavigation is a powerful tool to investigate the phase-dependent effects of neuronal oscillations on perception, cognition, and motor control.

\section{Introduction}

Evoked cortical responses show high intertrial variability that presumably depends to a great extent on intrinsic variations in neuronal excitability (Sadaghiani et al., 2010). Spontaneous fluctuations in neocortical excitability are not random but are organized into hierarchically nested oscillations of various frequencies, providing a precise temporal framework for information processing in the brain (Buzsáki, 2006). Neuronal oscillations are proposed to orchestrate the timing of information flow in a phase-dependent manner by

Received Sept. 20, 2011; revised 0ct. 16, 2011; accepted 0ct. 21, 2011.

Author contributions: T.O.B., M.M., L.M., J.B., and H.R.S. designed research; T.O.B., M.A.S., and C.L. performed research; M.M. contributed unpublished reagents/analytic tools; T.O.B., M.A.S., and C.L. analyzed data; T.O.B., M.M., L.M., J.B., and H.R.S. wrote the paper.

This work was funded by the Deutsche Forschungsgemeinschaft (Project A6, SFB 654 'Plasticity and Sleep'). T.O.B. was partially supported by the Dutch BrainGain Smart Mix Programme. H.R.S. was supported by a structural grant to NeurolmageNord (Bundesministerium für Bildung und Forschung Grant no. 01G0 0511) and a grant of excellence by the LundbeckFonden on the Control of Action "ContAct" (Grant no. R59 A5399).

Correspondence should be addressed to Dr. Til 0. Bergmann, Center for Cognitive Neuroimaging, Donders Institute for Brain, Cognition, and Behavior, Radboud University Nijmegen, P.O. Box 9101, 6500 HB Nijmegen, The Netherlands. E-mail: t.bergmann@donders.ru.nl.

DOI:10.1523/JNEUROSCI.4792-11.2012

Copyright $\odot 2012$ the authors $\quad 0270-6474 / 12 / 320243-11 \$ 15.00 / 0$ means of rhythmic input gain modulation, facilitating or suppressing synaptic input based on the neuron's current state of excitability (VanRullen and Koch, 2003; Buzsáki and Draguhn, 2004; Fries, 2005; Schroeder and Lakatos, 2009).

Rapid shifts in neocortical excitability (i.e., the extent or likelihood to which cortical neurons respond to synaptic input) likely depend on background changes in neocortical excitation (i.e., spontaneous neuronal activity at the population level). This can be probed by assessing the response to a stimulation as a function of ongoing brain activity. Indeed, human psychophysical and neuroimaging studies have shown that both the evoked cortical response to and the conscious perception of a sensory stimulus are modulated by very slow $(0.01-0.1 \mathrm{~Hz})$ fluctuations in neuronal excitation (Boly et al., 2007; Fox et al., 2007; Hesselmann et al., 2008; Monto et al., 2008; Sadaghiani et al., 2009) and variations in oscillatory power of alpha $(8-12 \mathrm{~Hz})$ and beta $(15-30$ $\mathrm{Hz}$ ) bands (Thut et al., 2006; van Dijk et al., 2008; van Ede et al., $2011)$, as well as the phase of slow $(0.1-1 \mathrm{~Hz})$, theta $(\sim 7 \mathrm{~Hz})$, and alpha oscillations (Kruglikov and Schiff, 2003; Massimini et al., 2003; Busch et al., 2009; Mathewson et al., 2009; Busch and VanRullen, 2010). 
A

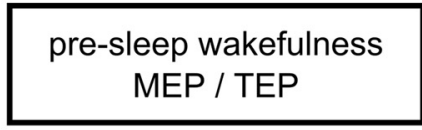

$\sim 15 \min$

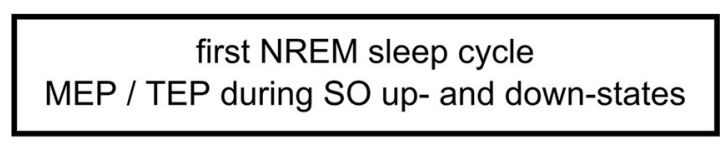

$\sim 75 \mathrm{~min}$ post-sleep wakefulness

MEP / TEP

$\sim 15 \min$

\section{B}

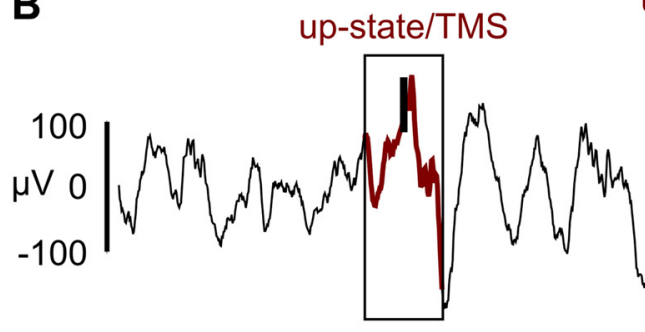

up-state/noTMS

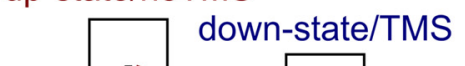

n-state/TMS

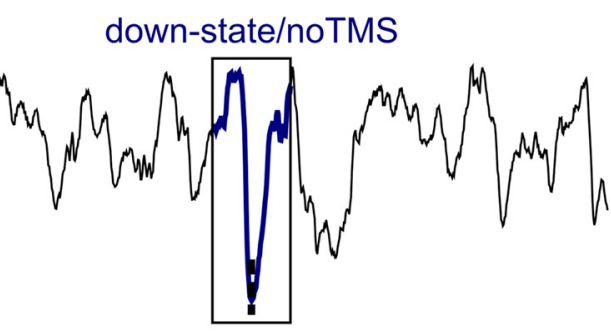

0
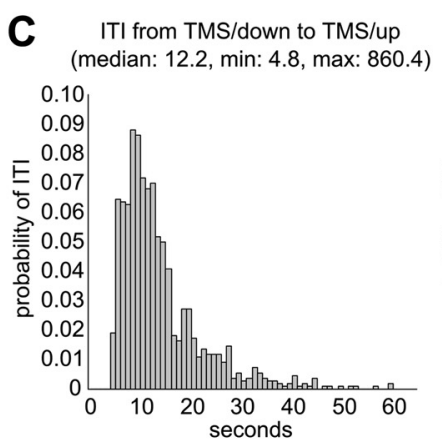

5

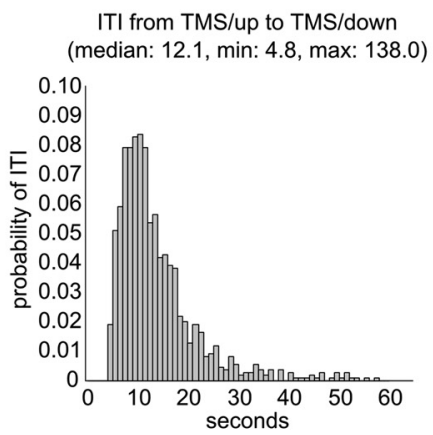

10

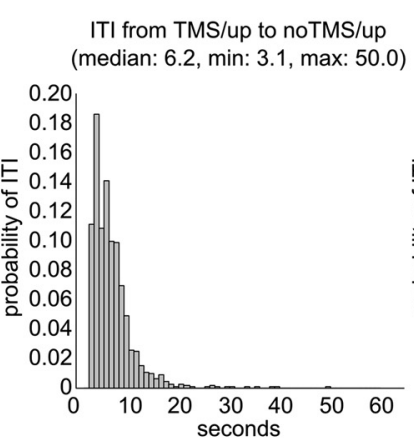

15 seconds

20

Figure 1. Experimental time line and example data. $A, M E P s$ (EMG at the contralateral hand) and TEPs (64-channel EEG) were acquired for SO up-and down-states during first NREM sleep cycle as well as during pre-sleep and post-sleep wakefulness. $\boldsymbol{B}$, An adaptive thresholding algorithm automatically detected up- and down-states of the slow oscillation during NREM sleep. TMS was triggered by every second event (TMS), whereas the others were left unstimulated as a baseline (noTMS). An example EEG data strip (channel (3) is shown containing all four event types. C, Histograms show relative frequencies ( $1 \mathrm{~s}$ bins) of the relevant intertrial intervals (ITI), and the corresponding descriptive statistics. Note that the few delays lasting even longer than $60 \mathrm{~s}$ are not included in the histograms for the sake of visibility. min, Minimum; max, maximum.

However, neocortical and thalamic contributions to the modulation of sensory-evoked responses are hard to disentangle. Transcranial magnetic stimulation (TMS) can directly probe the excitability of neocortical networks, as reflected for instance in fluctuations of EEG alpha activity, while bypassing the afferent sensory pathways that are subject to thalamic gating (Romei et al., 2008a, 2008b; Sauseng et al., 2009). In this study, we focused on the $<1 \mathrm{~Hz}$ neocortical slow oscillation (SO), which is the major organizing feature of non-rapid eye movement (NREM) sleep and the largest spontaneous fluctuation occurring under physiological conditions (Steriade et al., 1993; Achermann and Borbely, 1997). The SO shifts the membrane potential between phases of hyperpolarization (down-state), during which virtually all neocortical neurons are silent, and depolarization (up-state), which is associated with massive neuronal excitation. By grouping faster rhythms (spindles, gamma, and hippocampal ripples) into the up-state (Mölle et al., 2002, 2006; Sirota et al., 2003; Steriade, 2006), the SO is thought to orchestrate hippocampo-neocortical memory transfer during sleep (Diekelmann and Born, 2010).

We used a novel EEG-guided TMS approach to directly probe SO state-dependent shifts in neocortical excitability in sleeping subjects. Single-pulse TMS was used to stimulate the left motor cortical hand area $\left(\mathrm{Ml}_{\mathrm{HAND}}\right)$ selectively during either $\mathrm{SO}$ up- or down-states that were automatically detected in the ongoing EEG. Simultaneously measured motor-evoked potentials (MEPs) and TMS-evoked EEG potentials (TEPs) revealed that neocortical excitability is distinctly modulated by the actual state of the endogenous SO.

\section{Materials and Methods}

Participants. Twenty-three healthy volunteers participated in the experiments after giving written informed consent. Data are presented from 12 subjects ( 9 females; mean age, 24; range, 20-29 years) who were able to maintain slow-wave sleep (SWS) for a sufficient duration under the inconvenient recording conditions. Experimental procedures conformed to the Declaration of Helsinki and were approved by the Ethics Committee of the University of Kiel (Kiel, Germany). Participants were righthanded (Oldfield, 1971), free of medication, and had no history of neurological or psychiatric disease. They had to restrict their sleep to a maximum of $6 \mathrm{~h}$ during the previous night, ensuring moderate sleep pressure, and were not allowed to drink alcohol or caffeine or engage in excessive manual activities on the experimental day.

Experimental settings and procedure. Participants arrived in the laboratory at 8 P.M. and were prepared for sleep-TMS-EEG recordings. They were allowed to fall asleep around 10:30 P.M., slept for approximately the first sleep cycle, and then could leave the laboratory. Cortical excitability of the left $\mathrm{M} 1_{\mathrm{HAND}}$ was assessed by measuring MEPs and TEPs during SO up- and down-states during NREM sleep (Fig. 1). In addition, MEPs and TEPs were acquired during relaxed wakefulness shortly before (presleep) and after sleep (post-sleep). During wake recordings, subjects were instructed to keep their eyes open and focused on a fixation cross and not to blink in direct response to a TMS pulse (but after a second at the earliest). Subjects were bedded comfortably on a mattress of viscoelastic foam superimposed on a reclining chair in semi-horizontal position, wore light sleeping clothes, and were covered with a blanket. Their heads were stabilized with viscoelastic foam and two lateral fixations.

Transcranial magnetic stimulation. Single-pulse TMS of $M 1_{\mathrm{HAND}}$ was performed using a figure-of-eight shaped 'MC-B70' coil with an outer diameter of $100 \mathrm{~mm}$ connected to a MagPro-X100 stimulator (MagVen- 
ture). The magnetic stimulus had a biphasic pulse configuration (280 $\mu \mathrm{s}$ width). TMS was triggered by a CED Power 1401 control unit driven by Signal version 4.02 and Spike2 version 5.15 software during wakefulness and sleep, respectively (Cambridge Electronic Design). Recharging of capacitors was delayed by $1000 \mathrm{~ms}$ to prevent associated EEG artifacts directly following the TMS pulse.

The coil was positioned tangentially to the scalp above the left $\mathrm{Ml}_{\mathrm{HAND}}$ at an angle of $\sim 45^{\circ}$ to the sagittal plane, thus with the largest gradient inducing an electrical current in the brain tissue with posteriorlateral to anterior-medial direction roughly perpendicular to the central sulcus, as this is known to optimally evoke motor responses in the contralateral hand (Mills et al., 1992). The coil position at which stimuli at slightly suprathreshold intensity consistently yielded maximal MEPs in the contralateral first dorsal interosseus (FDI) muscle was considered the "motor hot spot" and was used for all measurements of a given subject. We used frameless stereotaxy (TMS-Navigator, Localite) based on a coregistered individual T1-weighted magnetic resonance (MR) image to register EEG electrode positions and to navigate the TMS coil. Spatial neuronavigation ensured that we maintained the optimal coil position throughout the experiment. MR images were acquired on a 3 tesla MR scanner (Achieva, Philips Medical Systems) using a standard MPRAGE sequence $\left(\mathrm{TR}=7.7 \mathrm{~ms} ; \mathrm{TE}=3.6 \mathrm{~ms}\right.$; flip angle $=8^{\circ} ; 170$ sagittal slices; $1 \times 1 \times 1 \mathrm{~mm}$ voxel size; field of view $=224 \times 224 \mathrm{~mm}$ ).

During wakefulness before and after sleep, we separately acquired 40 MEPs (interstimulus interval $=5 \mathrm{~s} ; 30 \%$ jitter; 3.3 min duration) and 200 TEPs (interstimulus interval $=3 \mathrm{~s} ; 30 \%$ jitter; 10 min duration). For MEP recordings, TMS intensity was adjusted to elicit mean peak-to-peak amplitudes around $1 \mathrm{mV}$ in the relaxed contralateral FDI muscle (47.2 \pm $7.5 \%$ maximum stimulator output), whereas TEPs were evoked at $90 \%$ resting motor threshold (Rossini et al., 1994) (36.5 $\pm 6.1 \%$ maximum stimulator output) according to a standard protocol (cf., Esser et al., 2006; Huber et al., 2008). During sleep, simultaneously acquired MEPs and TEPs were evoked with the same stimulation intensity as wake MEPs. The number of trials per subject depended on the number of onlinedetected slow oscillations but was always identical for SO up-states and down-states.

To avoid TMS click-related arousals, subjects were equipped with modified foam earplugs containing a silicon tube connected to headphones for delivering a continuous TMS-masking noise. For this purpose, TMS clicks were digitized and a continuous noise signal was composed of tightly packed TMS clicks superimposed on a background noise of phase-randomized frequencies from the spectral distribution of the TMS click. Sound volume was individually adjusted to optimally mask the TMS click without keeping subjects awake (Massimini et al., $2005,2007)$. In addition, a layer of air bubble film was placed between coil and EEG cap to attenuate bone conduction.

$E M G$ recordings. Surface EMG was recorded from the right FDI muscle with $\mathrm{Ag}-\mathrm{AgCl}$ skin electrodes using a bipolar belly-tendon montage. The raw EMG signals were amplified by 1000 (customized D360, Digitimer), filtered between 2 and $253 \mathrm{~Hz}$ (plus $50 \mathrm{~Hz}$ notch), and digitized at $5 \mathrm{kHz}$ per channel (CED Power 1401, Cambridge Electronic Design). The comparably low filter of $253 \mathrm{~Hz}$ was due to the customized hardware filter modification necessary for SO detection (see below, Temporal neuronavigation of TMS by online SO up- and down-state detection). EMG data were recorded with Signal and Spike2 software (Cambridge Electronic Design) during wakefulness and sleep, respectively.

EEG recordings. Sixty four-channel EEG (Fp1, Fp2, AF7, AF3, AFz, AF4, AF8, F7, F5, F3, F1, Fz, F2, F4, F6, F8, FT9, FT7, FC5, FC3, FC1, FC2, FC4, FC6, FT8, FT10, T7, C5, C3, C1, Cz, C2, C4, C6, T8, TP9, TP7, CP5, CP3, CP1, CPz, CP2, CP4, CP6, TP8, TP10, P7, P5, P3, P1, Pz, P2, P4, P6, $\mathrm{P} 8, \mathrm{PO} 7, \mathrm{PO} 3, \mathrm{POz}, \mathrm{PO} 4, \mathrm{PO} 8, \mathrm{O} 1, \mathrm{Oz}, \mathrm{O}_{2}$, and $2 \times$ physically linked earlobes; reference, FCz; ground, Fpz) was obtained via a customized Fast'n EasyCap electrode cap with TMS-compatible, extra flat $\mathrm{Ag}-\mathrm{AgCl}$ dot electrodes (EasyCap). Electrodes (despite C3; see below, Temporal neuronavigation of TMS by online SO up- and down-state detection) were connected to two battery-driven, TMS-compatible BrainAmp MR plus DC amplifiers (BrainProducts). Skin resistance at EEG electrodes was kept below $3 \mathrm{kOhm}$ by thorough skin preparation, and $\mathrm{Abralyt} \mathrm{HiCl}$ electrode paste (EasyCap) was used to ensure stable DC recordings throughout the entire experiment. Polysomnography further employed vertical and horizontal electrooculograms as well as an electromyogram at the chin (EMG) acquired via Ag-AgCl lens electrodes connected to a battery driven TMS-compatible BrainAmp ExG MR bipolar amplifier (BrainProducts). EEG data were recorded via BrainVision Recorder version 1.10 software (BrainProducts) with a resolution of $0.5 \mu \mathrm{V} / \mathrm{bit}$ at DC with a lowpass filter of $1 \mathrm{kHz}$ and digitized at a sampling rate of $5 \mathrm{kHz}$. EEG data acquisition was synchronized to the internal clock of the CED Power1401 triggering the TMS.

Temporal neuronavigation of TMS by online SO up- and down-state detection. EEG electrode $\mathrm{C} 3$ (near $\mathrm{M}_{\mathrm{HAND}}$ ) was physically referenced against linked earlobes amplified by 10,000 and bandpass filtered between 0.16 and $2 \mathrm{~Hz}$ using a customized bipolar amplifier (D360, Digitimer) with modified filter range and external trigger input for blocking/ unblocking. The signal was digitized at $5 \mathrm{kHz}$ (CED Power 1401, 16-bitADC; Cambridge Electronic Design) and used for online EEG analysis. Automatic detection of SO up- and down-states was achieved by an adaptive thresholding algorithm implemented in a Spike2 script that was controlling the built-in output sequencer of the CED Power1401 interface during data acquisition. By this means TMS was triggered without time lag, since the ongoing EEG signal of $\mathrm{C} 3$ was analyzed in real time on the hardware level of the CED Power 1401. SO up- and down-states were alternatingly detected based on certain threshold crossings. Default threshold criteria were $+35 \mu \mathrm{V}$ (rising flank) and $-75 \mu \mathrm{V}$ (falling flank) for SO up- and down states, respectively. These threshold criteria were updated every $0.5 \mathrm{~s}$ according to the minimum and maximum during the last $3 \mathrm{~s}$ of C3 EEG to account for general changes in SO amplitude when sleep deepens or lightens over time.

Every second online-detected up-/down-state triggered a TMS pulse. No TMS was given in the remaining half of detections, serving as nonstimulation baseline. EEG-guided TMS was started manually when online monitoring revealed stable NREM sleep and three or more SOs were detected within $30 \mathrm{~s}$. Stimulation intensity was increased stepwise to ensure that the discharge of the TMS coil did not wake up the subject. Note that only trials with target intensity were used for later analyses. Each time a TMS pulse was triggered, the bipolar amplifier was blocked for $1.2 \mathrm{~s}$ (from $-5 \mathrm{~ms}$ to $+1095 \mathrm{~ms}$ relative to the TMS pulse) to prevent persistent saturation of the bipolar EEG channel C3 by magneto-electric induction. Since blocking affected all channels, the amplifier was briefly unblocked between $+15 \mathrm{~ms}$ and $+95 \mathrm{~ms}$ to allow the acquisition of MEPs in the EMG channel. The EEG (but not the EMG) signal required a maximum of $1.5 \mathrm{~s}$ to fully recover from blocking/unblocking due to the low high-pass filter. Therefore, each detected SO was followed by a refractory period of $3.1 \mathrm{~s}$ ( $1.5 \mathrm{~s}$ for noTMS trials without blocking), which also ensured nonoverlapping data strips of at least $\pm 1.5 \mathrm{~s}$ around each event for later offline analyses. Note that EEG recordings for later offline EEG analyses and polysomnography were performed continuously using TMS-compatible hardware not requiring any blocking (BrainAmp MR plus and BrainAmp ExG MR; see above, EEG recordings). Importantly, due to the applied set of adaptive thresholding and timing criteria the algorithm detected merely a fraction of the actually present SO up- and down-states and did not result in a continuous stimulation in the SO frequency range during SWS but was much slower (Fig. 1 B and C).

Analysis of motor-evoked potentials. EMG data were analyzed using Signal version 4.02 and Spike2 version 5.15 software (Cambridge Electronic Design) and Matlab 7.7.0.471 (MathWorks). Wake and sleep peak-to-peak MEP amplitudes were calculated trialwise and then averaged per condition. Average MEP latencies (from TMS pulse to onset of first deflection) were calculated by considering only suprathreshold MEP $(>0.05 \mathrm{mV})$. Correlational analyses tested for a linear relationship between trial-to-trial variations in log-transformed MEP amplitudes and trial-to-trial variations in the SO potential at the time of stimulation (the data point directly preceding the TMS pulse) for every channel. Correlation coefficients were calculated separately for SO up- and down-states in every subject and were then tested channel-wise against zero on the group level (see below, Statistics).

Analysis of TMS-evoked potentials. EEG-data were analyzed using BrainVision Analyzer version 2 software (BrainProducts) and Matlab 7.7.0.471 (MathWorks). Linear interpolation between 0 and $25 \mathrm{~ms}$ rela- 
Table 1. MEP parameters for sleep SO up- and down-states as well as pre-sleep and post-sleep wakefulness measurements (mean \pm SD)

\begin{tabular}{|c|c|c|c|c|}
\hline & Up-state & Down-state & Pre-sleep & Post-sleep \\
\hline MEP amplitude (mV) & $0.65 \pm 0.31$ & $0.53 \pm 0.29$ & $1.00 \pm 0.63$ & $0.64 \pm 0.41$ \\
\hline Percent suprathreshold MEP (\%) & $92 \pm 10$ & $85 \pm 14$ & $99 \pm 20$ & $94 \pm 10$ \\
\hline Suprathreshold MEP amplitude (mV) & $0.70 \pm 0.31$ & $0.62 \pm 0.33$ & $1.01 \pm 0.63$ & $0.66 \pm 0.40$ \\
\hline MEP latency (ms) & $25.25 \pm 1.53$ & $25.65 \pm 1.86$ & $24.75 \pm 2.00$ & $24.77 \pm 1.98$ \\
\hline
\end{tabular}

tive to the TMS pulse eliminated TMS-related artifacts such as initial amplifier ringing $(\sim 0-7 \mathrm{~ms})$, head muscle potentials $(\sim 7-12 \mathrm{~ms})$, and exponential drifts due to electrode polarization (lasting for up to $\sim 25 \mathrm{~ms}$ in some channels), which would otherwise interfere with subsequent data processing (for recent technical reviews see Siebner et al., 2009a; Ilmoniemi and Kicić, 2010). Channel C3 (connected to the bipolar amplifier for SO detection), and where necessary bad channels (wake TEPs only) were topographically interpolated from neighboring electrodes (fourth order spherical splines). Data were re-referenced to linked mastoids, bandpass filtered between 0.16 and $100 \mathrm{~Hz}$ ( $48 \mathrm{~dB}$ /octave), and down-sampled to $250 \mathrm{~Hz}$. Sleep stages were visually scored based on electrode $\mathrm{C} 4$ ( $0.5-35 \mathrm{~Hz}$ bandpass filtered) according to standard criteria (Rechtschaffen and Kales, 1968).

For calculation of sleep TEPs, data were segmented from -1500 to +1500 ms around each TMS pulse (or noTMS event) and averaged per condition (up-TMS, down-TMS, up-noTMS, down-noTMS). Then, noTMS averages were subtracted from TMS averages (removing the underlying endogenous slow oscillation signal; compare Fig. $4 A$ ), and resulting TEPs were baseline-corrected ( -50 to $0 \mathrm{~ms}$ ). To account for interindividual variability of peak latencies, TEP amplitudes were individually determined for each subject as the local maximum $(\mathrm{P})$ or minimum $(\mathrm{N})$ in between the latencies of the two neighboring components (which were derived from the group signal average). Slow components (N400, P1000) were derived from a moderately filtered signal (see previous paragraph) and faster components (P40, N120, P180) from a $4 \mathrm{~Hz}$ (48 dB/octave) high-pass filtered signal enabling the assessment of amplitudes independent of the underlying slow potential. Differences between SO up- and down-state TEPs were then tested channel-wise on the group level (see Statistics).

Unlike for MEPs, no single-trial correlations of TEP with SO amplitude at the time of stimulation could be calculated because TEPs could not be reliably identified on the single-trial level against the background of high-amplitude endogenous SO activity. We therefore chose an alternative approach. Trials were sorted, separately for the four trial types (SO up-/down-state with/without TMS), according to the actual EEG potential in channel C5 directly preceding the TMS pulse (C5 was chosen because it revealed significant effects for both single-trial MEP correlations and all TEP SO up-state vs down-state comparisons; see Results). The four trial distributions were then each split into three bins of increasing SO up-state positivity (low, 0-33\%; middle, 33-66\%; high, 66$100 \%$ ) and decreasing SO down-state negativity (high, $100-66 \%$; middle, 66-33\%; low, 33-0\%), respectively. TEPs were calculated by subtraction of the respective noTMS averages, and the amplitudes of the individual P40 and N400 components were determined (as described above) for statistical comparisons. To relate TEPs and MEPs, we also calculated MEP amplitudes and the ratio of suprathreshold MEPs for the three bins.

For wake TEP, data were segmented from -50 to 1500 ms relative to the TMS pulse and baseline corrected ( -50 to $0 \mathrm{~ms}$ ). Trials with eye blinks were rejected semiautomatically whenever the averaged and $5 \mathrm{~Hz}$ low-pass filtered signal of channels FP1 and FP2 exceeded a peak-to-peak amplitude of $\pm 50 \mu \mathrm{V}$ within $200 \mathrm{~ms}$, and the remaining trials were averaged (pre-sleep, $128.1 \pm 42.2$; post-sleep, $136.7 \pm 43.7$; total, $264,8 \pm 80.5$ ). Amplitudes of the typical wake TEP components (P30, $\mathrm{N} 45, \mathrm{P} 60, \mathrm{~N} 100, \mathrm{P} 180, \mathrm{~N} 280$ ) were determined from the individual averages (in analogy to sleep TEP), and pre- and post-sleep measurements were compared channel-wise on the group level (see Statistics).

Analysis of TMS-induced oscillations. Continuous wavelet analyses were conducted on the $4 \mathrm{~Hz}$ high-pass filtered segments using complex Morlet wavelets (parameter $\mathrm{c}=10 ; 75$ linear $0.5 \mathrm{~Hz}$ steps from $3 \mathrm{~Hz}$ to 40
$\mathrm{Hz}$ ) with Garbor normalization, giving the instantaneous amplitude of the signal that was then averaged across trials. Again, averages based on the noTMS trials were subtracted from averages derived from the TMS trials to remove any oscillatory activity associated with the endogenous SO per se. Differences between SO up- and down-state TEPs were then tested channel-wise on the group level (see Statistics).

Statistics. If not stated otherwise, data are presented as mean \pm SD. Tests revealing a $p$ value below 0.05 were considered significant. MEP amplitudes and latencies were compared using one-sided paired $t$ tests testing the respective unidirectional hypotheses that motor cortical excitability is higher under the following conditions: (1) during SO upstates rather than down-states (see Introduction); (2) during wakefulness rather than NREM sleep (Grosse et al., 2002); and (3) before sleep rather than after sleep (Bergmann et al., 2008). MEP-SO single-trial correlations, as well as both sleep TEP (SO up-state $>$ SO down-state) and wake TEP (pre-sleep > post-sleep) amplitude differences (sleep: P40, N120, P180, N400, P1000; wake: P30, N45, P60, N100, P180, N280), were tested electrode-wise (i.e., mass univariate) using nonparametric permutation testing that included correction for multiple comparisons. Nonparametric permutation testing calculates $t$ values for all possible permutations of how experimental conditions could theoretically be allocated to empirical data points (i.e., correlation coefficients or difference values), generating the empirical $t$ distribution under the assumption of no effect (null hypothesis), and tests the actual $t$ value with respect to this distribution. Here, empirical $t$ distributions were first calculated electrode-wise, and correction for multiple comparisons was realized afterward by thresholding with the 95 th percentile of the maximum $t$ statistic calculated over all electrodes. $T$ values exceeding this threshold were thus considered significant at $p_{\text {corr }}<0.05$ (cf. Nichols and Holmes, 2002; Huber et al., 2004; Bergmann et al., 2008). All maps are generated by third-order spherical spline interpolation and scaled symmetrically with color codes in 24 steps ranging from blue (minimum) to red (maximum) values. For TMS-induced oscillation (TIO) differences between SO up- and downstates, $t$ values for channel-wise time-frequency matrices were calculated with clusters of $p<0.01$ considered significant (two-sided paired $t$ test).

\section{Results}

Subjects slept on average for $76.6 \pm 17.6 \mathrm{~min}$ (wake, $5.4 \pm 12.4$ min; stage 1, $5.4 \pm 4.6 \mathrm{~min}$; stage 2, $29.3 \pm 8.0 \mathrm{~min}$; SWS, $41.8 \pm$ $21.8 \mathrm{~min}$; sleep latency, $2.7 \pm 2.7 \mathrm{~min}$; SWS latency, $20.7 \pm 17.7$ $\mathrm{min}$ ) and $93 \pm 60$ trials were acquired per experimental condition (up-TMS, down-TMS, up-noTMS, down-noTMS). For half of the subjects the first valid TMS trial was delivered into the up-state and down-state, respectively. The intervals between two consecutive TMS trials (median, 12.1 s and $12.2 \mathrm{~s}$ for down-toup- and up-to-down-state, respectively), as well as TMS and noTMS trials (median, 6.2 and $6.9 \mathrm{~s}$ for up- and down-state, respectively) were sufficiently long and highly variable, rendering carryover effects between trials unlikely (Fig. 1C).

\section{Motor-evoked potentials}

On average, MEP amplitude was $~ 20 \%$ larger during SO upstates than during down-states $\left(t_{11}=4.18, p=0.0008\right.$; Table 1$)$. This difference was consistent across subjects, as 11 of the 12 subjects showed larger MEP amplitudes during the up-state of the SO (Fig. $2 \mathrm{~B}$ ). Single-pulse TMS also evoked more frequently a suprathreshold motor response (operationally defined as MEP 

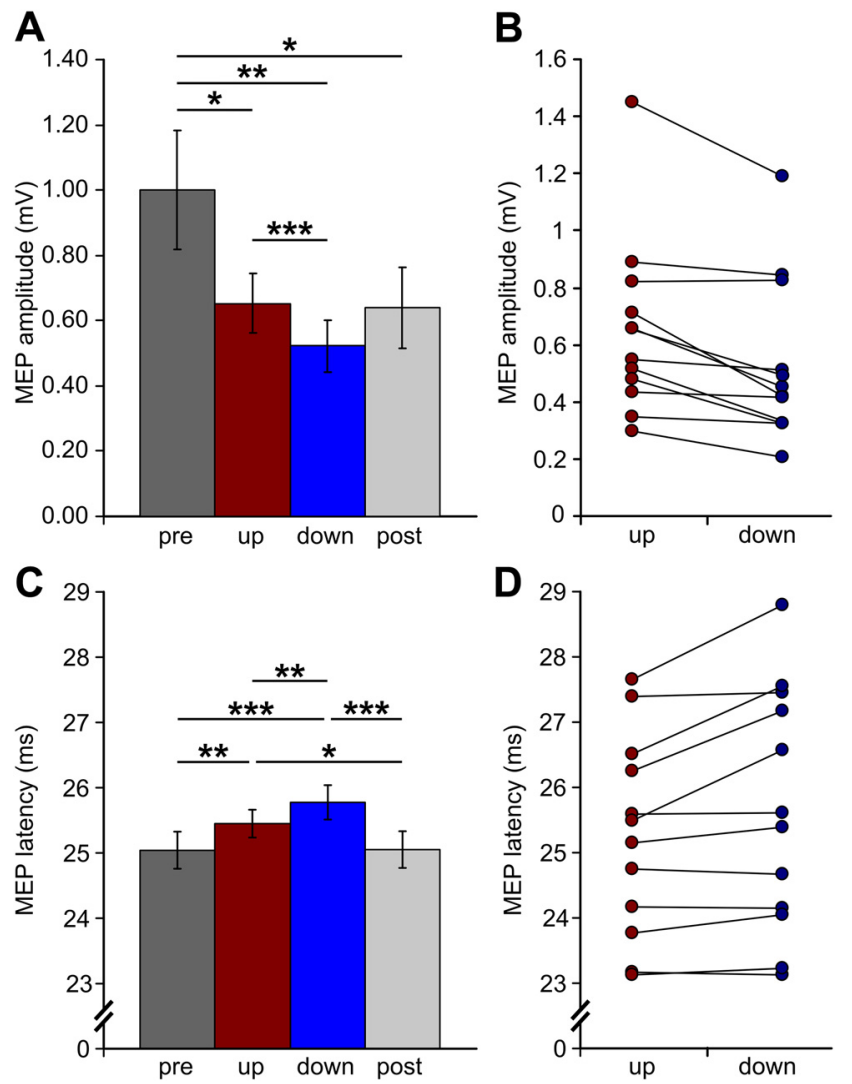

Figure 2. Motor-evoked potentials. $\boldsymbol{A}, \boldsymbol{C}$, Group averages of MEP amplitudes and latencies ( \pm SEM) for TMS stimulation during the up-state (red) and down-state (blue) of the sleep slow oscillation as well as for pre-sleep (dark gray) and post-sleep (light gray) wakefulness. Asterisks indicate significant comparisons: ${ }^{*} p<0.05,{ }^{* *} p<0.01,{ }^{* * *} p<0.001 . \boldsymbol{B}, \boldsymbol{D}$, Individual MEP amplitudes and latencies for $\mathrm{SO}$ up- and down-states for all subjects.

amplitude of $>0.05 \mathrm{mV}$ ) when given during up-states than during down-states $\left(t_{11}=3.09, p=0.005\right)$. When including only trials in which TMS actually evoked a suprathreshold MEP there was still a significant difference between states $\left(t_{11}=3.11, p=\right.$ $0.005)$, indicating that the effect is not based on threshold shifts alone. Also, MEP latencies were modified by the SO state, being shorter during up-states than during down-states $\left(t_{11}=2.83, p=\right.$ 0.008; Fig. 2C). A shorter MEP latency during SO up-states was present in 9 of 12 subjects (Fig. 2D).

Trial-to-trial variations in MEP amplitude correlated with trial-to-trial variations in SO amplitude at the time of stimulation. Although these correlations were relatively weak, with average $r$ values ranging from 0.13 to 0.18 , they were consistent across subjects $\left(p_{\text {corr }}<0.05\right)$ and topographically specific for the site of stimulation (Fig. 3): MEP amplitudes were positively related to the absolute EEG amplitude value for both SO up-states (at electrodes C5, CP5, T7, FT7, and TP7) and SO down-states (at electrodes $\mathrm{C} 3, \mathrm{C} 5, \mathrm{CP} 1, \mathrm{CP} 3, \mathrm{CP} 5, \mathrm{P} 3, \mathrm{P} 5$, and P7). That is, for the left sensorimotor cortex, the more pronounced (depolarized) the upstate the larger the MEP amplitude, and the more pronounced (hyperpolarized) the down-state the smaller the MEP amplitude. This relationship is corroborated by the mean comparisons of MEP amplitudes for different levels of SO up-state positivity ( middle $>$ low: $t_{11}=3.03, p=0.006$; high $>$ low: $\mathrm{t}_{11}=2.35, p=$ 0.02; Fig. $6 G$ ) and SO down-state negativity (high $<$ middle: $t_{11}$ $=1.89, p=0.04$; high $<$ low: $t_{11}=2.51, p=0.01$; Fig. $\left.6 \mathrm{H}\right)$ at channel C5. A similar effect was also revealed when comparing the mean ratio of suprathreshold MEPs for different levels of SO up-state positivity (middle $>$ low: $t_{11}=2.32, p=0.02$; high $>$ low: $t_{11}=1.81, p=0.049$; Fig. $6 I$ ) and SO down-state negativity (high $<$ low: $t_{11}=3.27, p=0.004$; high $<$ middle: $t_{11}=3.12, p=$ 0.005; Fig. $6 J$ ). Therefore, the more positive the up-state or the less negative the down-state, the larger the MEP amplitudes, and more suprathreshold MEPs were evoked.

The SO state-dependent modulation of the MEP was observed on top of a more general decrease in MEP amplitude by $41 \%$ relative to pre-sleep wakefulness (Fig. $2 A$ ) which was evident for both SO up-states $\left(t_{11}=2.05, p=0.03\right)$ and down-states $\left(t_{11}=\right.$ $2.80, p=0.008)$. Interestingly, MEP amplitudes remained lower during the post-sleep measurement (post-sleep vs pre-sleep: $t_{11}$ $=1.92, p=0.04$ ). Likewise, MEP latencies were generally prolonged during sleep relative to both pre- and post-sleep wakefulness (pre-sleep vs up-state: $t_{11}=2.78, p=0.009$; pre-sleep vs down-state: $t_{11}=4.83, p=0.0003$; post-sleep vs up-state: $t_{11}=$ 2.34, $p=0.02$; post-sleep vs down-state: $t_{11}=4.43, p=0.0005$; Fig. $2 C$ ), although latencies did not differ between pre- and postsleep measurements $(p>0.4)$.

\section{TMS-evoked potentials}

TEPs were superimposed on the endogenous slow oscillation from which they were disentangled by subtraction of the noTMS average (Fig. 4). As visible from the group average (Figs. 4, 5A), responses evoked during SO up- and down-states were qualitatively highly comparable, showing the same pattern of alternating positive and negative deflections that are referred to in the following as P40, N120, P180, N400, and P1000 (Fig. 5A). Scalp current source density maps (Fig. $5 B$ ) suggested that the early fast TEP components (P40, N120) are located in the stimulated left sensorimotor cortex (but note also the contralateral $\mathrm{M} 1_{\text {HAND }}$ ), with the P180 already extending into more fronto-central sites (P180). The subsequent slow components (N400, P1000) had a strong frontal peak, also accompanied by lateral (N400) or medial (P1000) central peaks in the sensorimotor cortex. However, as indicated by the group average, TEPs evoked during the SO up-state were larger than those evoked during the down-state. Comparisons of individual TEP component amplitudes revealed significantly larger amplitudes $\left(p_{\text {corr }}<0.05\right)$ for the P40 peak (FC3, FC5, C3, C5, CP3, CP5), the N120 peak (F1, C5), and the N400 peak (35 mostly fronto-central and centro-parietal channels; Fig. 5C and Table 2). Moreover, within both SO states the TEP amplitude depended on the actual level of up-state positivity and down-state negativity at C5, respectively. The N400 component was larger for more positive $\mathrm{SO}$ up-states (high $>$ low: $t_{11}=$ 5.05, $p=0.0002$; high $>$ middle: $t_{11}=2.10, p=0.03$; Fig. $6 E$ ) and smaller for more negative SO down-states (high $<$ low: $t_{11}=$ 2.40, $p=0.02$; middle $<$ low: $t_{11}=1.95, p=0.04$; Fig. $6 F$ ). A less pronounced effect was also evident for the P40 component in both SO up-states (middle $>$ low: $t_{11}=2.34, p=0.02$; Fig. $6 C$ ) and down-states (high $<$ low: $t_{11}=3.02, p=0.006$; Fig. $6 D$ ).

TEPs evoked during wakefulness showed a strikingly different pattern compared to those during NREM sleep. They were of faster frequencies, lower amplitudes, and shorter duration, consisting of the well described components P30, N45, P60, N100, P180, and N280 (Figs. 4B, 5A). Pre- and post-sleep TEPs did not differ significantly in any of their components for any channel $\left(p_{\text {corr }}>0.1\right.$; Table 2$)$.

\section{TMS-induced oscillations}

Wavelet analyses revealed TMS-induced increases in spindle activity $(\sim 12-16 \mathrm{~Hz})$ during the late positive deflection of the TEP, i.e., the P1000 component (Fig. 7A). Interestingly, spindle activ- 
A

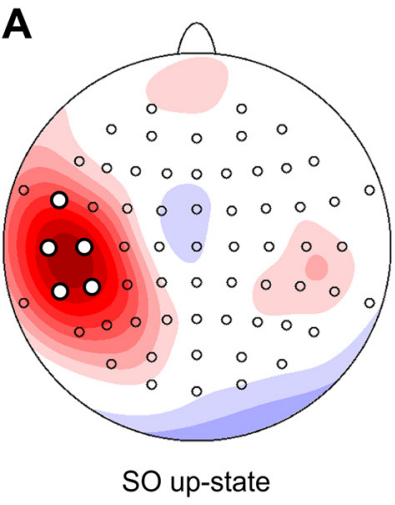

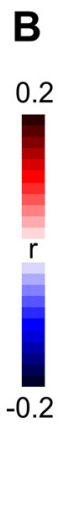
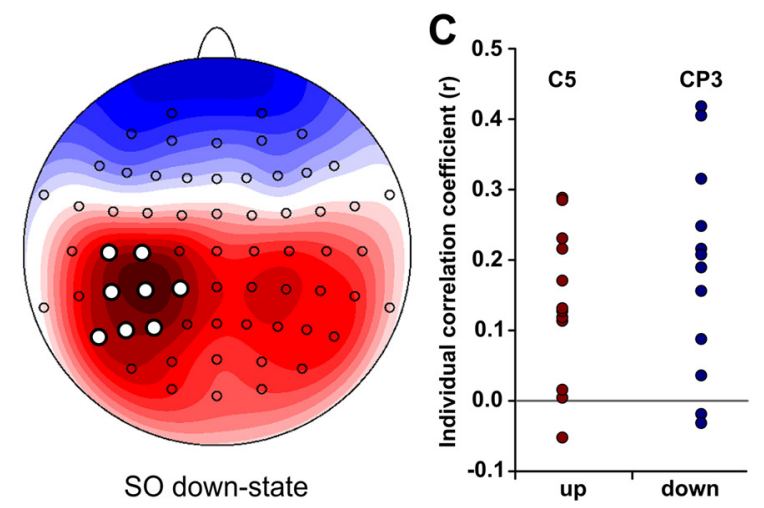

Figure 3. $\boldsymbol{A}, \boldsymbol{B}$, Single-trial correlations between SO amplitude (directly before TMS) and MEP amplitude (log transformed) for $\mathrm{SO}$ up-states ( $\boldsymbol{A}$ ) and $\mathrm{SO}$ down-states ( $\boldsymbol{B}$ ). MEP amplitude depended on the current EEG potential in the left sensorimotor cortex. The more depolarized the larger the MEP (up-state) and the more hyperpolarized, the smaller the MEP (down-state). Colors represent group-averaged correlation coefficients ranging from 0.2 (red) to -0.2 (blue) for each electrode. White dots indicate correlations significant at $p_{\text {corr }}<0.05$. C, Individual correlation coefficients for peak electrodes C5 (up-state) and CP3 (down-state).

A
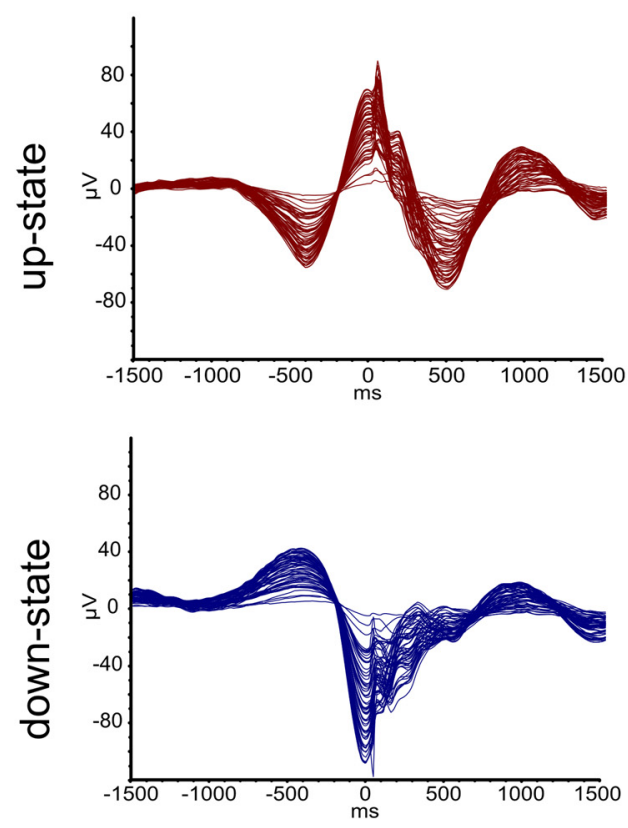

B

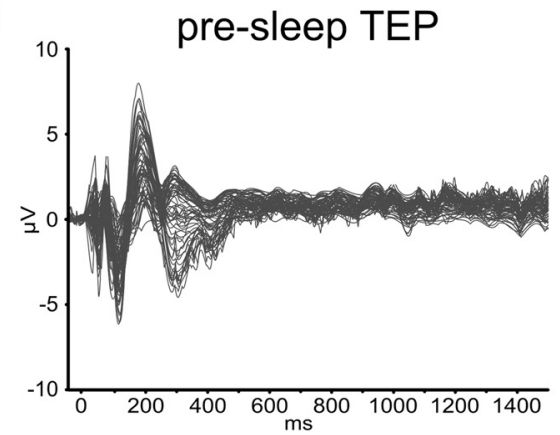

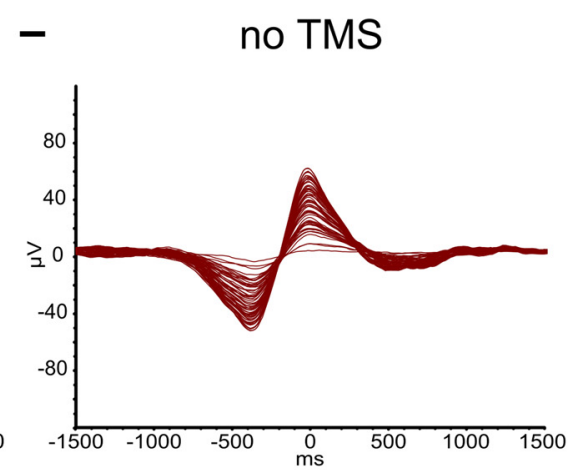

$=\quad$ TEP
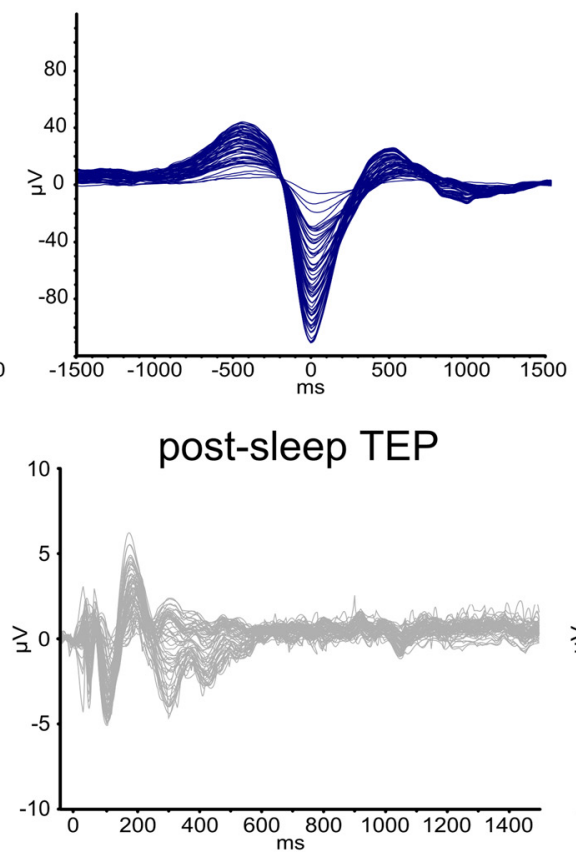
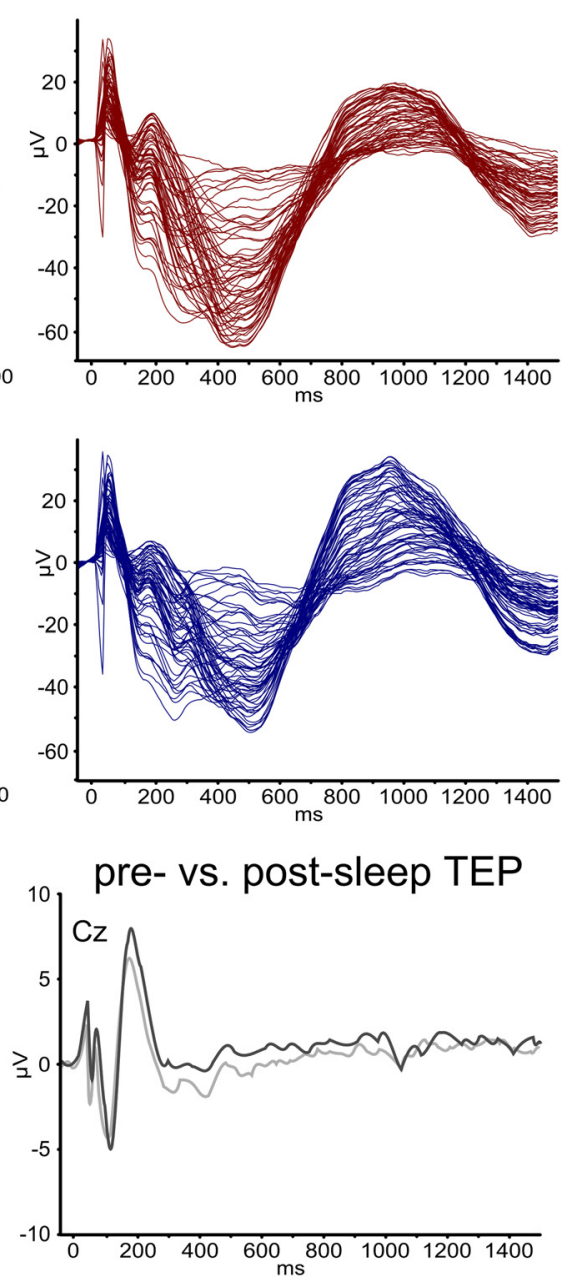

Figure 4. Butterfly diagrams. A, Grand averages of detected slow oscillations for all experimental conditions and calculation of TEPs. The figure shows butterfly diagrams (all channels superimposed; TMS artifacts removed) time locked to the TMS-pulse (at $0 \mathrm{~ms}$ ) for $\mathrm{SO}$ up-states (top row, red) and $\mathrm{SO}$ down-states (bottom row, blue) that had either been stimulated (TMS, left column) or merely detected (noTMS, middle column), as well as the respective subtraction of both, i.e., the actual response to TMS without the underlying endogenous oscillation (TEP, right column). Note that scaling is increased for the latter. B, Grand average TEPs from pre-sleep (left, dark gray) and post-sleep (middle panel, light gray) measurements as well as a superposition of representative channel $\mathrm{C}$ for both (right panel). Wake TEPs are of much smaller amplitude than sleep TEPs (different scaling), but note the different stimulation intensities as well (see Materials and Methods). 


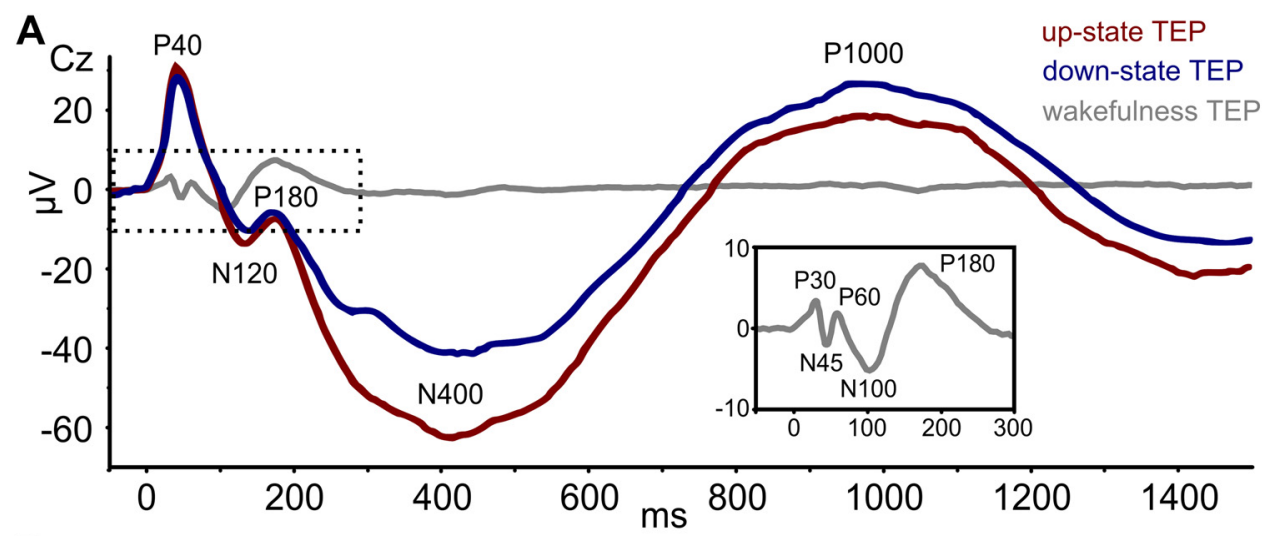

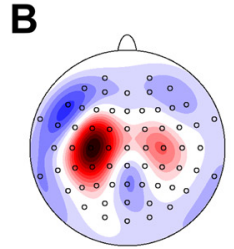

P40

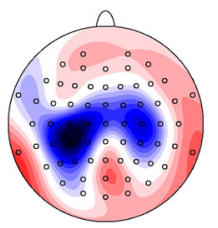

N120

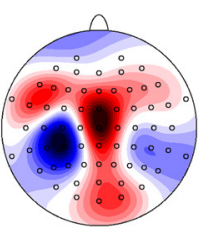

P180

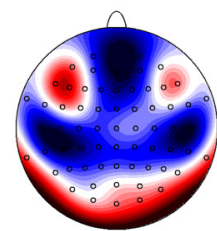

N400

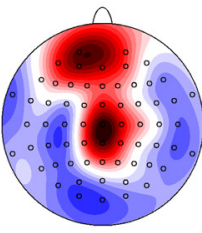

P1000

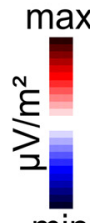

$\min$
C

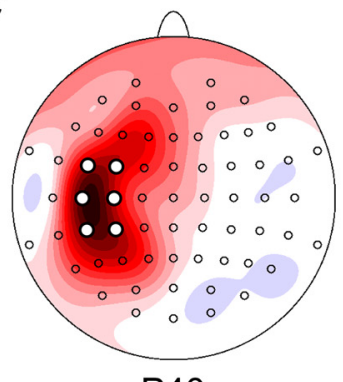

P40

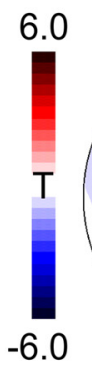

$-6.0$

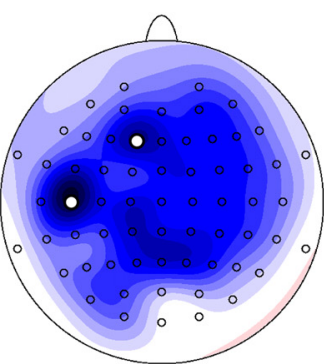

N120

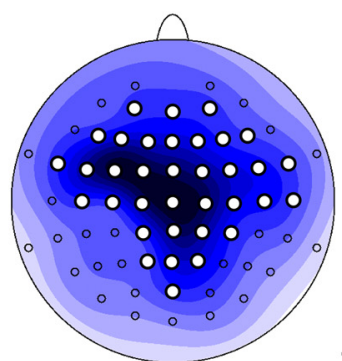

$\mathrm{N} 400$

9.0

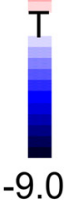

Figure 5. TMS-evoked potentials. $A$, Group-averaged TEPs in representative channel Cz for TMS stimulation during SO up-states (red), SO down-states (blue), and wakefulness (gray). The wake TEP is shown for visual comparison with the inset depicting an enlarged version of the dotted area for better visibility (averaged across pre-sleep and post-sleep measures). TEP components are labeled according to their polarity and approximate latency. Note the striking difference between the SO-like sleep TEPs and the typical high-frequency wake TEP. $\boldsymbol{B}$, Scalp current source density (CSD) maps for the most pronounced sleep TEP components (averaged across S0 states; high-pass filtered for P40, N120, and P180). Maps are scaled individually according to their respective minimum (blue) and maximum (red) CSD values $\left(\mu \mathrm{V} / \mathrm{m}^{2}\right)$. C, Statistical maps depict color-coded $t$ values from electrode-wise amplitude comparisons ( $\mathrm{S} 0$ up-state minus down-state) of individual TEP components. White dots indicate differences significant at $p_{\text {corr }}<0.05$ (no significant channels were revealed for P180 and P1000). See Materials and Methods for details.

Table 2. TEP amplitudes (in $\mu \mathrm{V}$ ) for sleep SO for up- and down-states as well as pre- and post-sleep wakefulness measurements for representative channels $\mathrm{C} 3$, $\mathrm{C5}$, and $\mathrm{Cz}$ in the vicinity of the stimulation site (mean \pm SD)

\begin{tabular}{|c|c|c|c|c|c|c|}
\hline & \multicolumn{3}{|l|}{ Up-state } & \multicolumn{3}{|l|}{ Down-state } \\
\hline & C3 & $C 5$ & $\mathrm{Cz}$ & C3 & C5 & $\mathrm{Cz}$ \\
\hline P40 & $27.99 \pm 10.51$ & $20.22 \pm 6.93$ & $22.84 \pm 8.01$ & $24.56 \pm 10.78$ & $17.57 \pm 7.91$ & $21.20 \pm 8.33$ \\
\hline N120 & $-15.27 \pm 4.33$ & $-11.75 \pm 3.78$ & $-14.28 \pm 3.23$ & $-12.65 \pm 5.21$ & $-9.53 \pm 4.05$ & $-11.61 \pm 3.67$ \\
\hline P180 & $5.51 \pm 3.98$ & $4.40 \pm 2.42$ & $9.18 \pm 6.52$ & $4.13 \pm 3.28$ & $3.40 \pm 2.46$ & $6.88 \pm 5.48$ \\
\hline N400 & $-69.48 \pm 21.85$ & $-57.81 \pm 18.74$ & $-76.36 \pm 21.77$ & $-49.63 \pm 21.93$ & $-42.73 \pm 19.14$ & $-49.41 \pm 18.13$ \\
\hline \multirow[t]{3}{*}{ P1000 } & $15.30 \pm 12.84$ & $9.84 \pm 8.37$ & $27.18 \pm 17.03$ & $21.24 \pm 14.96$ & $16.02 \pm 11.54$ & $32.24 \pm 17.18$ \\
\hline & \multicolumn{3}{|l|}{ Pre-sleep } & \multicolumn{3}{|l|}{ Post-sleep } \\
\hline & $\mathrm{C} 3$ & $\mathrm{C5}$ & $\mathrm{Cz}$ & $\mathrm{C} 3$ & $\mathrm{C5}$ & $\mathrm{Cz}$ \\
\hline P30 & $1.5 \pm 3.77$ & $2.38 \pm 2.04$ & $4.38 \pm 2.71$ & $0.67 \pm 4.36$ & $3.29 \pm 3.29$ & $3.17 \pm 3.27$ \\
\hline N45 & $-4.83 \pm 9.47$ & $-1.33 \pm 2.63$ & $-1.96 \pm 2.85$ & $-4.83 \pm 7.18$ & $-0.38 \pm 3.61$ & $-3.33 \pm 3.10$ \\
\hline P60 & $2.38 \pm 2.95$ & $2.54 \pm 2.47$ & $3.96 \pm 4.17$ & $2.08 \pm 3.37$ & $3.46 \pm 2.83$ & $2.58 \pm 5.04$ \\
\hline N100 & $-5.71 \pm 4.18$ & $-6.38 \pm 3.44$ & $-6.79 \pm 4.21$ & $-7.00 \pm 4.42$ & $-7.38 \pm 3.85$ & $-6.54 \pm 2.65$ \\
\hline P180 & $6.67 \pm 3.43$ & $4.92 \pm 2.29$ & $9.13 \pm 5.12$ & $6.25 \pm 2.57$ & $4.96 \pm 2.32$ & $7.96 \pm 3.57$ \\
\hline $\mathrm{N} 280$ & $-1.96 \pm 2.54$ & $-1.88 \pm 2.20$ & $-2.17 \pm 3.44$ & $-4.04 \pm 4.40$ & $-2.83 \pm 4.15$ & $-3.08 \pm 3.04$ \\
\hline
\end{tabular}


A
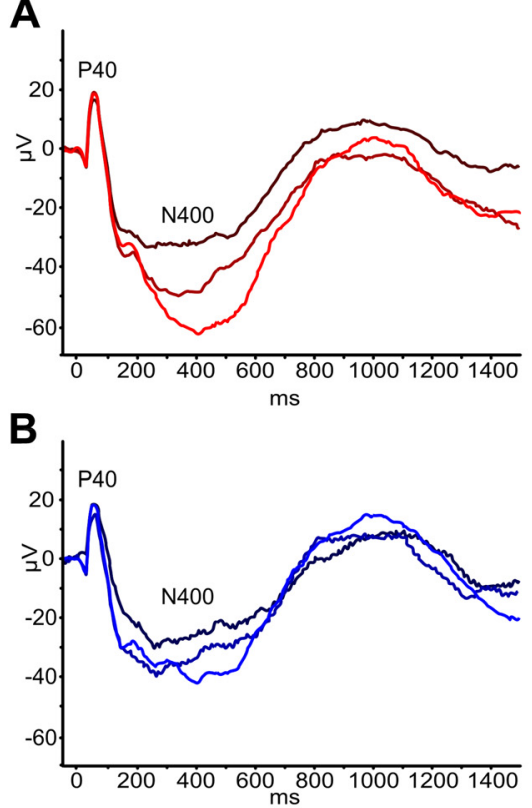

C

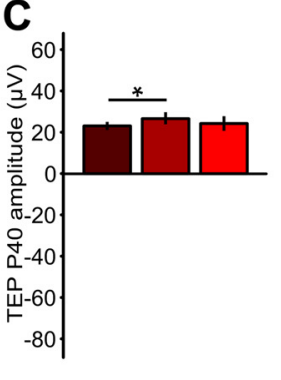

D

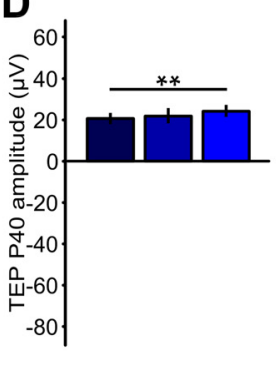

E

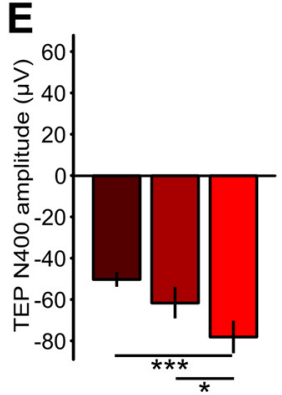

F
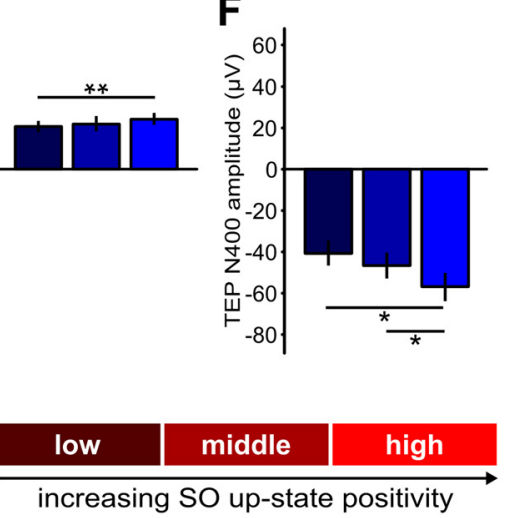

G
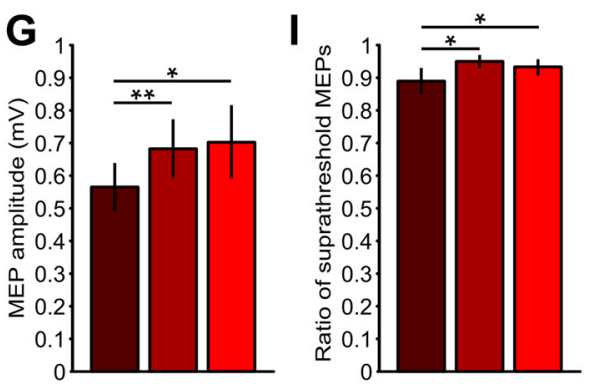

H
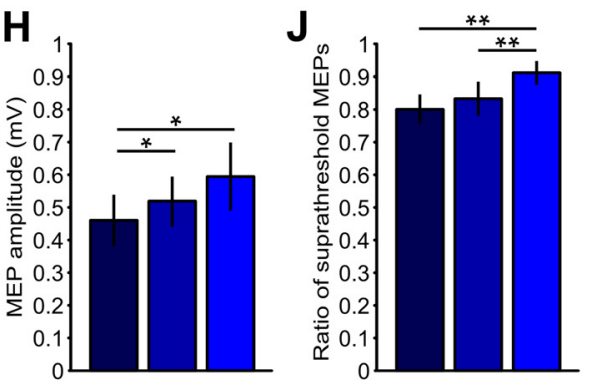

\section{high}

$\underset{\text { decreasing SO down-state negativity }}{\longrightarrow}$

Figure 6. TEPs and MEPs depend on the actual SO potential at channel $C 5$ at the time of stimulation. Trials were binned into three different levels of $S 0$ up-state positivity (red shades) and $S 0$ down-state negativity (blue shades), respectively (see Materials and Methods). $\boldsymbol{A}, \boldsymbol{B}$, Grand average TEP waveforms. Bar charts depict average ( \pm SEM) C, D, TEP P40 amplitudes. E, $\boldsymbol{F}$, TEP N400 amplitudes. $\mathbf{G}, \boldsymbol{H}$, MEP amplitudes. $\boldsymbol{I}, \boldsymbol{J}$, Ratios of suprathreshold MEPs. TEP and MEP amplitudes were individually determined (see Materials and Methods; compare Fig. 5) and averaged per bin. Asterisks indicate significant comparisons: ${ }^{*} p<0.05,{ }^{* *} p<0.01,{ }^{* * *} p<0.001$.

ity was stronger when TMS was applied during the up-state compared to the down-state of the endogenous SO (Fig. 7B). Stimulation during the up-state also resulted generally in stronger oscillatory activity in other frequency bands like theta $(4-8$ $\mathrm{Hz})$, alpha/slow spindle $(8-12)$, beta $(16-30 \mathrm{~Hz})$, and low gamma $(>30 \mathrm{~Hz})$.

\section{Discussion}

Temporal neuronavigation of TMS revealed rapid changes in the responsiveness of neocortical neurons in the human $\mathrm{M} 1_{\mathrm{HAND}}$ during the sleep slow oscillation. Single-pulse TMS elicited MEPs with larger amplitudes and shorter latencies during up-states than during down-states. In addition, TMS during up-states produced SO-like TEPs with larger early (P40) and late (N400) components and induced oscillations with larger amplitude in various frequency bands, particularly in the spindle range. On a trial-by-trial basis, MEP amplitude increased linearly with SO up-state positivity and decreased linearly with SO down-state negativity, and TEP amplitudes also depended on the actual SO potential within each state. Despite this clear SO state dependency, however, neither MEPs nor TEPs resembled wake-like responses during the up-state.

\section{Sleep-dependent changes in neocortical excitability}

Our findings tie in well with previous TMS studies on sleepdependent changes in neocortical excitability. The decrease in MEP amplitude and prolongation of MEP latency during NREM sleep relative to pre-sleep wakefulness have previously been observed to accompany the progression from wakefulness to deep sleep (Grosse et al., 2002; Salih et al., 2005; Avesani et al., 2008). While these changes might be partially due to a general suppression of spinal motor neurons during sleep, increased paired- pulse intracortical inhibition suggests a cortical contribution as well (Salih et al., 2005; Avesani et al., 2008). This sleep-dependent suppression might be also reflected by the reduced MEP amplitude after sleep, an effect previously observed following a full night of sleep (Bergmann et al., 2008). Several mechanisms might contribute to this post-sleep reduction such as synaptic downscaling (Tononi and Cirelli, 2006), depression by limb immobilization (Huber et al., 2006), or possibly sleep inertia (Bertini et al., 2004), and the present study was not designed to distinguish them. The fact that we found no significant pre-sleep to postsleep changes in wake TEP amplitude (although the group signal averages in Fig. $4 B$ suggest a slight reduction after sleep) might render a spinal effect more likely. However, it is still possible that a TEP effect (reflecting cortical mechanisms) would be visible after longer sleep periods or when able to analyze earlier components ( $<25 \mathrm{~ms}$ ) that were compromised by TMS-related artifacts in the current study.

Regardless of SO state, TEP evoked at $\mathrm{M}_{\text {HAND }}$ during NREM sleep differed markedly from the typical TEP pattern (P30, N45, P60, N100, P180, and N280) that we and others have observed during wakefulness. Wake TEPs are characterized by a faster frequency, lower amplitude, and shorter duration (Paus et al., 2001; Bonato et al., 2006; Siebner et al., 2009a). In contrast, the sleep TEP during both SO up- and down-states rather resembled an endogenous SO, which is in good agreement with previously reported TEP patterns evoked at dorsal sensorimotor regions during NREM sleep (Massimini et al., 2007): A strong local response at the stimulation site (P40) was followed by a high amplitude slow potential (N400, P1000) with widespread topography and a fronto-central peak, grouping sleep spindles in its "up-state" similar to the cortical response to electrical stimulation in rat frontal 
A

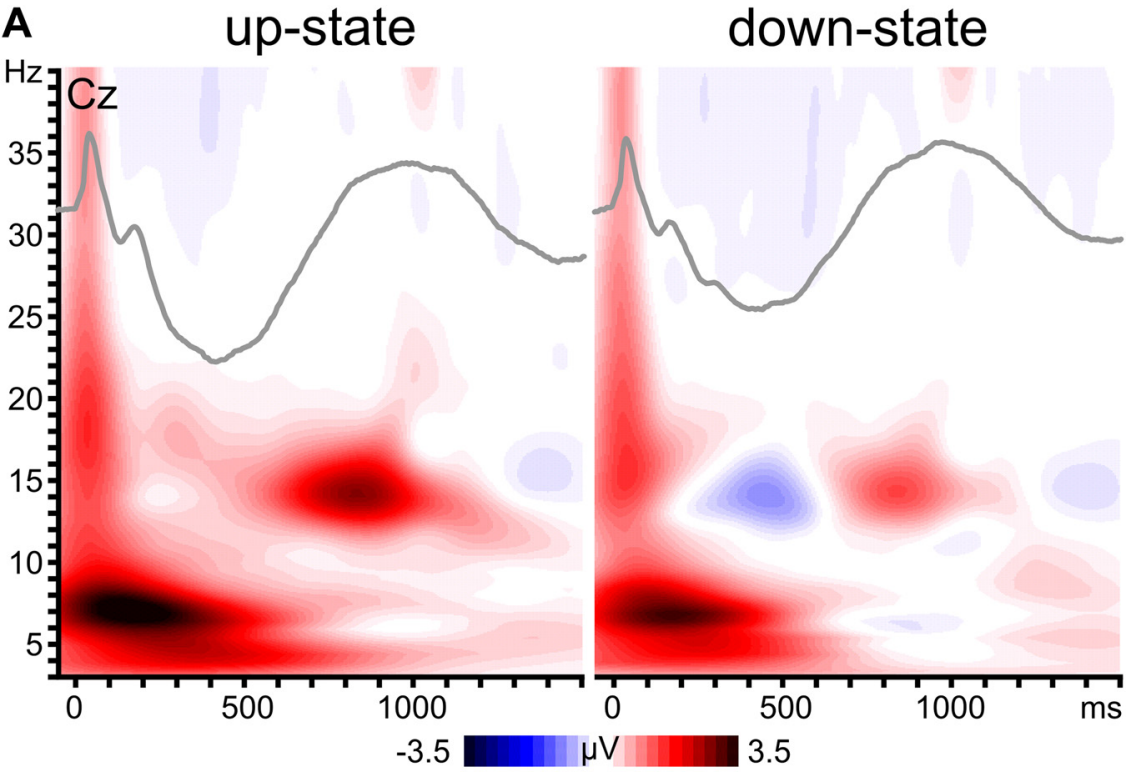

B
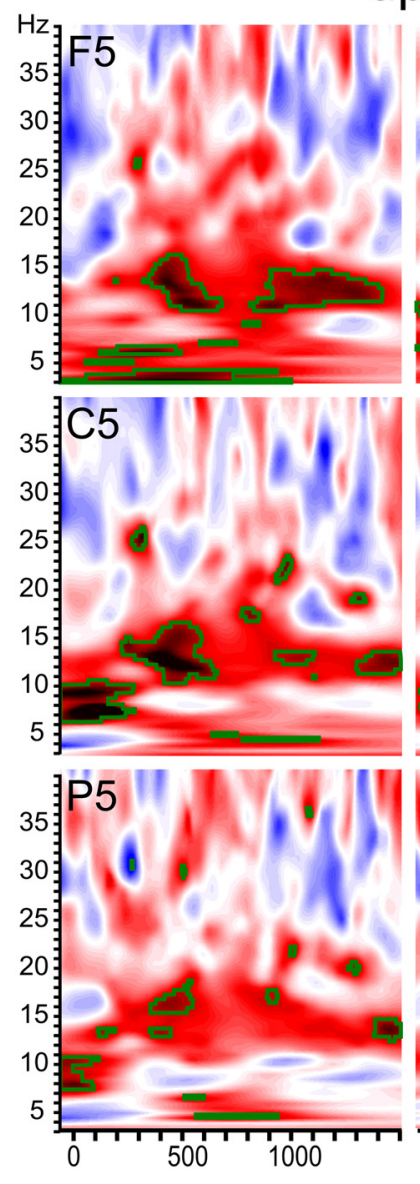

up- vs. down-state
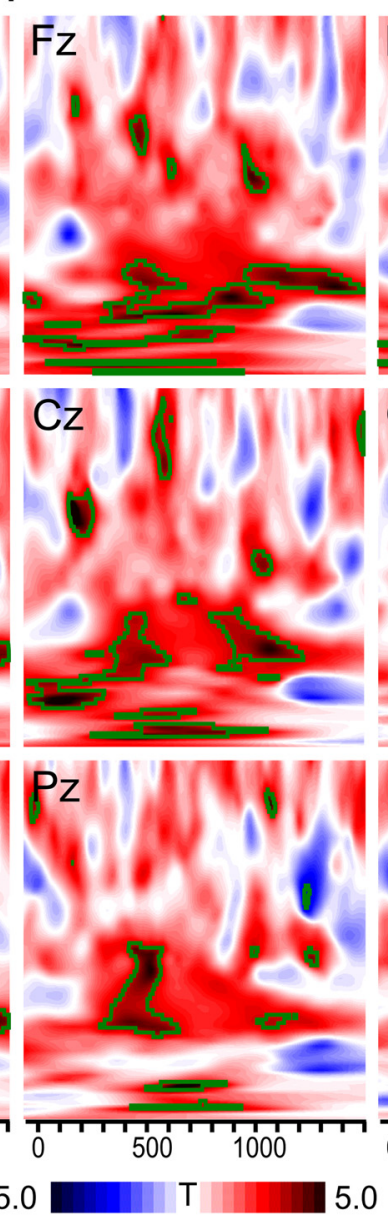
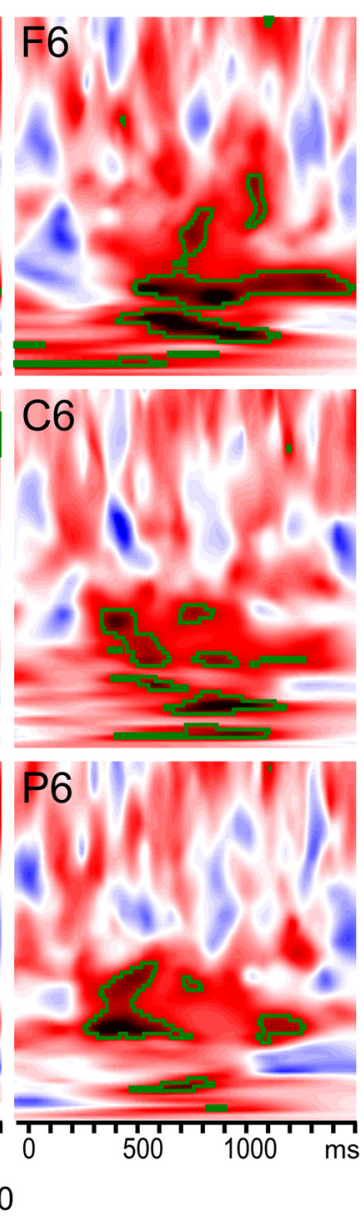

Figure 7. TMS-induced oscillations. $\boldsymbol{A}$, Group averaged time-frequency plots (instantaneous amplitude) depict TIOs after subtraction of the endogenous $\mathrm{SO}$ for $\mathrm{SO}$ up- and down-states in representative channel $\mathrm{Cz}$. Inserted TEPs (gray lines) illustrate the relation to the TEP time course. Note that spindle activity $(12-16 \mathrm{~Hz})$ is grouped within the positive phase of the evoked slow potential. $\boldsymbol{B}$, Time-frequency plots (statistical $t$ values) contrasting both states for a representative selection of channels. Green outlines indicate significant clusters ( $p<0.01$, two-sided).

cortex and parietal cortex (Vyazovskiy et al., 2009). Of note, TMS of other cortical sites along the midline (Massimini et al., 2007) or the dorsal premotor cortex (Massimini et al., 2005) did not evoke the same SO-like response, but nevertheless TEPs with larger amplitudes and markedly reduced temporospatial complexity when compared to wakefulness or REM sleep (Massimini et al., 2010). This reduced complexity has been interpreted as a breakdown of effective corticocortical connectivity, causing the loss of consciousness during sleep (Tononi and Massimini, 2008).

\section{SO state-dependent modulation of neocortical excitability}

Temporally neuronavigated TMS with combined TEP and MEP measurements enabled us to directly probe, for the first time, neocortical responsiveness during human SO up- and down-states. The relationship between MEP amplitude and SO state might correspond to a basic finding for TMS of $\mathrm{M1}_{\mathrm{HAND}}$ : enhancing prestimulus motor cortical excitation by voluntary contraction of the corresponding hand muscles (or merely the preparation or imagination of contraction) results in immediate amplification of MEP amplitude and reduction of MEP latency, because neurons are brought closer to firing threshold (Siebner et al., 2009b). Spontaneous fluctuations in the membrane potential of neocortical motor neurons during the $\mathrm{SO}$ might have a similar effect. Besides the difference in excitability between $\mathrm{SO}$ states, the observed linear relationship between local SO potential and MEP amplitude within each state further supports this notion. These correlations were relatively weak. Yet it is remarkable that its predictive value was still evident and, in addition, topographically specific for the site of stimulation considering that the surface EEG potential at the electrode level is not specific for $\mathrm{M} 1_{\mathrm{HAND}}$ motor neurons but sums over vast neuron populations. Given that SO potential variance was severely limited by the applied threshold criterion, the actual correlation is likely to be much larger. The within-state dependency of TEP amplitude on the actual level of SO potential at the stimulation site further supports the interpretation that cortical excitability is gradually modulated by the ongoing SO. However, whether the effect on TEPs is mainly driven by a modulation of response magnitude or response likelihood cannot be disentangled based on the current data, although MEP results suggest an effect on both.

One previous human EEG study has shown that the SO modulates the ampli- 
tude of somatosensory-evoked potential (SEP) in response to peripheral nerve stimulation using a post hoc trial sorting approach (Massimini et al., 2003). In contrast to the statedependent modulation of MEP and TEP amplitudes, SEP amplitudes were smallest during the $\mathrm{SO}$ up-state and largest during the SO down-state (Massimini et al., 2003). However, a direct comparison of the two studies is difficult, as SEP amplitudes are likely to be modulated by both thalamic and cortical excitability fluctuations. Intracellular recordings in the anesthetized cat revealed that SO state-dependent EPSPs in neocortical neurons differ for electrical stimulation of pre-thalamic sensory afferents and corticocortical projections (Timofeev et al., 1996). Following pre-thalamic stimulation, cortical EPSPs were reduced during the up-state and completely abolished during the down-state because SO-related hyperpolarization of thalamocortical neurons reduced signal transmission to the cortex. In contrast, EPSPs following cortical stimulation were not diminished during the SO but were negatively related to prestimulus membrane potential, i.e., larger EPSP amplitudes were observed for more hyperpolarized membrane potentials (presumably due to the larger distance from firing threshold). Importantly, however, only up-state EPSPs resulted in action potentials (Timofeev et al., 1996), which might partially explain the positive linear relationship between MEP amplitude and prestimulation EEG potential, because MEP amplitude rather depends on the number of corticospinal motor neurons firing action potentials than on EPSP amplitude.

\section{Complementary "read outs" of neocortical responsiveness}

TMS of $M 1_{\text {HAND }}$ primarily excites neocortical motor neurons trans-synaptically via the depolarization of axonal fibers (Di Lazzaro et al., 2004). Each TMS pulse therefore generates a highly synchronized presynaptic input to the neocortical output neurons that is subject to input gain modulation. We simultaneously employed complementary "read-outs" of neocortical responsiveness. Whereas MEPs reliably indicate the excitability of corticospinal motor neurons in $\mathrm{Ml}_{\mathrm{HAND}}$ with good topographical specificity, TEPs and TIOs rather reflect the temporospatial spread of neocortical activity and reflect the integration of recurrent activity in large scale corticocortical and corticothalamic circuits (Ilmoniemi and Kicić, 2010; Siebner et al., 2009a). Since we found all measures to coherently depend on the SO state, it appears likely that the SO effectively modulates the gain of synaptic input at the level of neocortical neurons, which in turn results in different corticospinal as well as corticocortical and corticothalamic output levels.

\section{Temporal neuronavigation of TMS based on online EEG}

We used an adaptive online thresholding algorithm operationalizing SO up- and down-states as sufficiently large positive- or negative-going deflections in the $0.16-2 \mathrm{~Hz}$ filtered EEG signal in the immediate vicinity of the target site. Despite this comparably coarse criterion, the resulting signal averages closely resembled those resulting from more sophisticated offline $\mathrm{SO}$ analyses (e.g., Mölle et al., 2002). This EEG-triggered TMS approach enabled us to record the same number of unstimulated SO control events based on identical criteria, providing an optimal tool for disentangling spontaneous and evoked activity by subtracting respective averages (cf. Kruglikov and Schiff, 2003). It further ensured a sufficient number of trials for the actual cortical states of interest, which is more difficult to achieve by randomized stimulation and post hoc trial sorting.

\section{Conclusion}

The demonstration of rapid fluctuations in neocortical responsiveness during the up- and down-states of the sleep SO corroborates the notion that neocortical oscillations cause a rhythmic modulation of the cortical input-gain function. Jointly oscillating neuron assemblies not only increase their impact on downstream neurons by synchronous firing but also modulate their responsiveness to synaptic input from upstream sites, thereby amplifying or suppressing information flow depending on the current oscillatory phase (Buzsáki and Draguhn, 2004; Fries, 2005, 2009; Schroeder and Lakatos, 2009). In fact, this temporal segregation into windows of preferred neuronal processing or "duty cycles" is a powerful means to orchestrate neuronal processing in cognitive functions like perception, attention, learning, or memory (VanRullen and Koch, 2003; Diekelmann and Born, 2010; Sauseng et al., 2010).

In addition, our results demonstrate the feasibility of temporally neuronavigated TMS based on online EEG to perform statedependent brain stimulation. This novel approach is highly relevant to systems neuroscience (Hartmann et al., 2011; Jensen et al., 2011), as it opens up new possibilities to noninvasively probe the causal contribution of distinct cortical oscillations to specific aspects of perception, cognition, and motor control.

\section{References}

Achermann P, Borbély AA (1997) Low-frequency $(<1 \mathrm{~Hz})$ oscillations in the human sleep electroencephalogram. Neuroscience 81:213-222.

Avesani M, Formaggio E, Fuggetta G, Fiaschi A, Manganotti P (2008) Corticospinal excitability in human subjects during nonrapid eye movement sleep: single and paired-pulse transcranial magnetic stimulation study. Exp Brain Res 187:17-23.

Bergmann TO, Mölle M, Marshall L, Kaya-Yildiz L, Born J, Siebner RH (2008) A local signature of LTP- and LTD-like plasticity in human NREM sleep. Eur J Neurosci 27:2241-2249.

Bertini M, Ferrara M, De Gennaro L, Curcio G, Fratello F, Romei V, Pauri F, Rossini PM (2004) Corticospinal excitability and sleep: a motor threshold assessment by transcranial magnetic stimulation after awakenings from REM and NREM sleep. J Sleep Res 13:31-36.

Boly M, Balteau E, Schnakers C, Degueldre C, Moonen G, Luxen A, Phillips C, Peigneux P, Maquet P, Laureys S (2007) Baseline brain activity fluctuations predict somatosensory perception in humans. Proc Natl Acad Sci U S A 104:12187-12192.

Bonato C, Miniussi C, Rossini PM (2006) Transcranial magnetic stimulation and cortical evoked potentials: a TMS/EEG co-registration study. Clin Neurophysiol 117:1699-1707

Busch NA, VanRullen R (2010) Spontaneous EEG oscillations reveal periodic sampling of visual attention. Proc Natl Acad Sci USA 107:16048-16053.

Busch NA, Dubois J, VanRullen R (2009) The phase of ongoing EEG oscillations predicts visual perception. J Neurosci 29:7869-7876.

Buzsáki G (2006) Rhythms of the brain. New York: Oxford UP.

Buzsáki G, Draguhn A (2004) Neuronal oscillations in cortical networks. Science 304:1926-1929.

Diekelmann S, Born J (2010) The memory function of sleep. Nat Rev Neurosci 11:114-126.

Di Lazzaro V, Oliviero A, Pilato F, Saturno E, Dileone M, Mazzone P, Insola A, Tonali PA, Rothwell JC (2004) The physiological basis of transcranial motor cortex stimulation in conscious humans. Clin Neurophysiol 115:255-266.

Esser SK, Huber R, Massimini M, Peterson MJ, Ferrarelli F, Tononi G (2006) A direct demonstration of cortical LTP in humans: a combined TMS/EEG study. Brain Res Bull 69:86-94.

Fox MD, Snyder AZ, Vincent JL, Raichle ME (2007) Intrinsic fluctuations within cortical systems account for intertrial variability in human behavior. Neuron 56:171-184.

Fries P (2005) A mechanism for cognitive dynamics: neuronal communication through neuronal coherence. Trends Cogn Sci 9:474-480.

Fries P (2009) Neuronal gamma-band synchronization as a fundamental process in cortical computation. Annu Rev Neurosci 32:209-224. 
Grosse P, Khatami R, Salih F, Kühn A, Meyer BU (2002) Corticospinal excitability in human sleep as assessed by transcranial magnetic stimulation. Neurology 59:1988-1991.

Hartmann T, Schulz H, Weisz N (2011) Probing of brain states in real-time: Introducing the ConSole environment. Front Psychol 2:36.

Hesselmann G, Kell CA, Eger E, Kleinschmidt A (2008) Spontaneous local variations in ongoing neural activity bias perceptual decisions. Proc Natl Acad Sci U S A 105:10984-10989.

Huber R, Ghilardi MF, Massimini M, Tononi G (2004) Local sleep and learning. Nature 430:78-81.

Huber R, Ghilardi MF, Massimini M, Ferrarelli F, Riedner BA, Peterson MJ, Tononi G (2006) Arm immobilization causes cortical plastic changes and locally decreases sleep slow wave activity. Nat Neurosci 9:1169-1176.

Huber R, Määttä S, Esser SK, Sarasso S, Ferrarelli F, Watson A, Ferreri F, Peterson MJ, Tononi G (2008) Measures of cortical plasticity after transcranial paired associative stimulation predict changes in electroencephalogram slow-wave activity during subsequent sleep. J Neurosci 28:7911-7918.

Ilmoniemi RJ, Kicić D (2010) Methodology for combined TMS and EEG. Brain Topogr 22:233-248.

Jensen O, Bahramisharif A, Oostenveld R, Klanke S, Hadjipapas A, Okazaki YO, van Gerven MA (2011) Using brain-computer interfaces and brainstate dependent stimulation as a tool in cognitive neuroscience. Front Psychol 2:100.

Kruglikov SY, Schiff SJ (2003) Interplay of electroencephalogram phase and auditory-evoked neural activity. J Neurosci 23:10122-10127.

Massimini M, Rosanova M, Mariotti M (2003) EEG slow (approximately 1 $\mathrm{Hz}$ ) waves are associated with nonstationarity of thalamo-cortical sensory processing in the sleeping human. J Neurophysiol 89:1205-1213.

Massimini M, Ferrarelli F, Huber R, Esser SK, Singh H, Tononi G (2005) Breakdown of cortical effective connectivity during sleep. Science 309:2228-2232.

Massimini M, Ferrarelli F, Esser SK, Riedner BA, Huber R, Murphy M, Peterson MJ, Tononi G (2007) Triggering sleep slow waves by transcranial magnetic stimulation. Proc Natl Acad Sci U S A 104:8496-8501.

Massimini M, Ferrarelli F, Murphy M, Huber R, Riedner B, Casarotto S, Tononi G (2010) Cortical reactivity and effective connectivity during REM sleep in humans. Cogn Neurosci 1:176-183.

Mathewson KE, Gratton G, Fabiani M, Beck DM, Ro T (2009) To see or not to see: prestimulus alpha phase predicts visual awareness. J Neurosci 29:2725-2732.

Mills KR, Boniface SJ, Schubert M (1992) Magnetic brain stimulation with a double coil: the importance of coil orientation. Electroencephalogr Clin Neurophysiol 85:17-21.

Mölle M, Marshall L, Gais S, Born J (2002) Grouping of spindle activity during slow oscillations in human non-rapid eye movement sleep. J Neurosci 22:10941-10947.

Mölle M, Yeshenko O, Marshall L, Sara SJ, Born J (2006) Hippocampal sharp wave-ripples linked to slow oscillations in rat slow-wave sleep. J Neurophysiol 96:62-70.

Monto S, Palva S, Voipio J, Palva JM (2008) Very slow EEG fluctuations predict the dynamics of stimulus detection and oscillation amplitudes in humans. J Neurosci 28:8268-8272.

Nichols TE, Holmes AP (2002) Nonparametric permutation tests for functional neuroimaging: a primer with examples. Hum Brain Mapp 15:1-25.

Oldfield RC (1971) The assessment and analysis of handedness: the Edinburgh inventory. Neuropsychologia 9:97-113.

Paus T, Sipila PK, Strafella AP (2001) Synchronization of neuronal activity in the human primary motor cortex by transcranial magnetic stimulation: an EEG study. J Neurophysiol 86:1983-1990.

Rechtschaffen A, Kales A (1968) A manual of standardized terminology, techniques and scoring system for sleep stages of human subjects. Washington, DC: United States Government Printing Office.

Romei V, Rihs T, Brodbeck V, Thut G (2008a) Resting electroencephalogram alpha-power over posterior sites indexes baseline visual cortex excitability. Neuroreport 19:203-208.

Romei V, Brodbeck V, Michel C, Amedi A, Pascual-Leone A, Thut G (2008b)
Spontaneous fluctuations in posterior alpha-band EEG activity reflect variability in excitability of human visual areas. Cereb Cortex 18:2010-2018.

Rossini PM, Barker AT, Berardelli A, Caramia MD, Caruso G, Cracco RQ, Dimitrijević MR, Hallett M, Katayama Y, Lücking CH (1994) Noninvasive electrical and magnetic stimulation of the brain, spinal cord and roots: basic principles and procedures for routine clinical application. Report of an IFCN committee. Electroencephalogr Clin Neurophysiol 91:79-92.

Sadaghiani S, Hesselmann G, Kleinschmidt A (2009) Distributed and antagonistic contributions of ongoing activity fluctuations to auditory stimulus detection. J Neurosci 29:13410-13417.

Sadaghiani S, Hesselmann G, Friston KJ, Kleinschmidt A (2010) The relation of ongoing brain activity, evoked neural responses, and cognition. Frontiers in systems neuroscience 4:20.

Salih F, Khatami R, Steinheimer S, Hummel O, Kühn A, Grosse P (2005) Inhibitory and excitatory intracortical circuits across the human sleepwake cycle using paired-pulse transcranial magnetic stimulation. J Physiol 565:695-701.

Sauseng P, Klimesch W, Gerloff C, Hummel FC (2009) Spontaneous locally restricted EEG alpha activity determines cortical excitability in the motor cortex. Neuropsychologia 47:284-288.

Sauseng P, Griesmayr B, Freunberger R, Klimesch W (2010) Control mechanisms in working memory: a possible function of EEG theta oscillations. Neurosci Biobehav Rev 34:1015-1022.

Schroeder CE, Lakatos P (2009) Low-frequency neuronal oscillations as instruments of sensory selection. Trends Neurosci 32:9-18.

Siebner HR, Bergmann TO, Bestmann S, Massimini M, Johansen-Berg H, Mochizuki H, Bohning DE, Boorman ED, Groppa S, Miniussi C, PascualLeone A, Huber R, Taylor PC, Ilmoniemi RJ, De Gennaro L, Strafella AP, Kähkönen S, Klöppel S, Frisoni GB, George MS, et al. (2009a) Consensus paper: combining transcranial stimulation with neuroimaging Brain Stimul 2:58-80.

Siebner HR, Hartwigsen G, Kassuba T, Rothwell JC (2009b) How does transcranial magnetic stimulation modify neuronal activity in the brain? Implications for studies of cognition. Cortex 45:1035-1042.

Sirota A, Csicsvari J, Buhl D, Buzsáki G (2003) Communication between neocortex and hippocampus during sleep in rodents. Proc Natl Acad Sci U S A 100:2065-2069.

Steriade M (2006) Grouping of brain rhythms in corticothalamic systems. Neuroscience 137:1087-1106.

Steriade M, Nuñez A, Amzica F (1993) A novel slow ( $<1 \mathrm{~Hz}$ ) oscillation of neocortical neurons in vivo: depolarizing and hyperpolarizing components. J Neurosci 13:3252-3265.

Thut G, Nietzel A, Brandt SA, Pascual-Leone A (2006) Alpha-band electroencephalographic activity over occipital cortex indexes visuospatial attention bias and predicts visual target detection. J Neurosci 26:9494-9502.

Timofeev I, Contreras D, Steriade M (1996) Synaptic responsiveness of cortical and thalamic neurones during various phases of slow sleep oscillation in cat. J Physiol 494:265-278.

Tononi G, Cirelli C (2006) Sleep function and synaptic homeostasis. Sleep Med Rev 10:49-62.

Tononi G, Massimini M (2008) Why does consciousness fade in early sleep? Ann N Y Acad Sci 1129:330-334.

van Dijk H, Schoffelen JM, Oostenveld R, Jensen O (2008) Prestimulus oscillatory activity in the alpha band predicts visual discrimination ability. J Neurosci 28:1816-1823.

van Ede F, de Lange F, Jensen O, Maris E (2011) Orienting attention to an upcoming tactile event involves a spatially and temporally specific modulation of sensorimotor alpha- and Beta-band oscillations. J Neurosci 31:2016-2024.

VanRullen R, Koch C (2003) Is perception discrete or continuous? Trends Cogn Sci 7:207-213.

Vyazovskiy VV, Faraguna U, Cirelli C, Tononi G (2009) Triggering slow waves during NREM sleep in the rat by intracortical electrical stimulation: effects of sleep/wake history and background activity. J Neurophysiol 101:1921-1931. 